\title{
Association of autoimmune diseases with celiac disease and its risk factors
}

\author{
Yusuf Kayar ${ }^{1}$, Ramazan Dertli²
}

\begin{abstract}
Objectives: Autoimmune Diseases (AIDs) are detected in celiac patients. Our purpose was to determine the AIDs that are associated with celiac disease and related risk factors in the Turkish population.

Methods: The study included 230 celiac patients who were diagnosed and followed-up in our clinics between 2015-2019. All AIDs that accompanied the celiac disease were recorded, and their association with risk factors was analyzed.

Results: The mean age of the patients was $35.6 \pm 10.6$ years (age range:18-72). A total of $58(25.2 \%)$ patients were male and the mean age at onset of disease was $29.1 \pm 6.5$ years. The duration of disease follow-up was $6.5 \pm 4.6$ years. One hundred and twenty two $(53 \%)$ patients were on a strict diet, and $72(31.3 \%)$ patients had accompanying AID. Hashimoto thyroiditis was found in $39(17 \%)$ patients, asthma in $16(7 \%)$ patients as the most common comorbidities. There was a significant relation between AID and female gender, age of diagnosis being $<40$ years, duration of disease, non-GIS symptoms at the time of admission, and nonemployment status.

Conclusion: Screening other AIDs in celiac patients are important, especially in individuals who have risk factors. Considering that many AIDs may develop in the future despite dietary compliance, patients should be followed with a multidisciplinary approach.
\end{abstract}

KEYWORDS: Autoimmune Diseases, Celiac Disease, Risk Factors.

doi: https://doi.org/10.12669/pjms.35.6.821

How to cite this:

Kayar Y, Dertli R. Association of autoimmune diseases with celiac disease and its risk factors. Pak J Med Sci. 2019;35(6):1548-1553. doi: https://doi.org/10.12669/pjms.35.6.821

This is an Open Access article distributed under the terms of the Creative Commons Attribution License (http://creativecommons.org/licenses/by/3.0), which permits unrestricted use, distribution, and reproduction in any medium, provided the original work is properly cited.

\section{INTRODUCTION}

Celiac Disease (CD) is an autoimmune enteropathy based on gluten intolerance in genetically predisposed individuals, and is characterized by inflammatory process resulting in flattening of

1. Yusuf Kayar,

2. Ramazan Dertli,

1,2: Department of Internal Diseases, Division of Gastroenterology,

Van Education and Research Hospital,

Van, Turkey.

Correspondence:

Yusuf Kayar,

Department of Gastroenterology,

Van Education and Research Hospital,

65100, Van, Turkey.

Email: ykayar@yahoo.com

* Received for Publication:

* Revision Received:

* Revision Accepted:

April 1, 2019

August 31, 2019

September 11, 2019 the villus, which occur in the small intestine, and loss of absorption function. ${ }^{1,2}$ As it is the case in other autoimmune diseases (AIDs), the immune response against antigens results in the production of autoantibodies and tissue destruction in CD. ${ }^{3}$ The disease is related closely to genes encoding HLADQ2 and HLADQ8. ${ }^{3}$

It was reported in previous reports that; there is a significant increase in the prevalence of AIDs like Insulin-Dependent Diabetes Mellitus (IDDM), thyroid diseases, connective tissue diseases, skin diseases, autoimmune hepatitis and primary biliary cirrhosis in celiac patients. ${ }^{4}$ This rate was found to be $34 \%$ for the first time in the study that was conducted by Ventura et al. with 909 celiac patients, and it was argued that long-term exposure to gluten triggered the development of AIDs. ${ }^{5}$ In this respect, in a study in which 703 patients who had CD were evaluated in Finland, it was determined that the 
rate of AIDs was 31\%; and in a study that was conducted in France, 924 patients were analyzed, and the rate of AIDs was reported as $19.3 \% .{ }^{6,7} \mathrm{It}$ was argued that this might share a pathogenic basis that included the same environmental triggers, genetic disposition, impaired intestinal barrier, and increased permeability in the intestine.,

Although contradictory results were reported in previous studies, it was argued that there is a significant relation between AIDs development and some risk factors in celiac patients. ${ }^{6,7}$ In the literature, there are studies showing that; there are significant relations between the presence of AIDs in the family, diagnosis of CD in early decades, duration of gluten exposure, presence of female gender, presence of non-gastrointestinal system (GIS) symptoms, and development of AIDs. ${ }^{6,7}$ In some studies that were conducted by considering the development of AIDs as secondary to prolonged gluten exposure, it was shown that serum insulin-related antibody and anti-thyroid antibody levels were reduced at a significant level in the follow-up of children who were fed without gluten; however, in some other studies it was reported that a gluten-free diet did not prevent the development of AIDs. ${ }^{9,10}$ In the present study, the purpose was to determine the AIDs accompanying the $\mathrm{CD}$ in Turkish population; and to investigate the risk factors affecting the development of AIDs.

\section{METHODS}

Subjects: The patients who were diagnosed and followed-up in the clinics of Gastroenterology were included in the present study. The celiac patients whose records were included in the hospital database between 2015 and 2019, and newlydiagnosed celiac patients in our outpatient clinics were included in the study. For all patients who were diagnosed with $\mathrm{CD}$, the inclusion criteria for the study were having positive results of the Antibody level test (Anti-Endomysium and Tissue Transglutaminase Antibody (anti-TTG)), which is carried out on clinical and laboratory suspicion, and having consistent results of the tissue samples taken in endoscopy in histopathological examination according to the Marsh classification. The patients who did not continue follow-ups in our clinic, whose data could not be obtained or who had missing data and who were pregnant were not included in the study. A total of 230 patients with $\mathrm{CD}$, whose ages varied between 18 and 72 years, whose data were complete, and who continued their follow-up regularly, were included in the study. Written informed consent forms were received from all participants.

Evaluation of Immunological, Demographic and Clinic Features: The demographic and anthropometric characteristics (age, gender, onset of disease, duration of disease, presence of $\mathrm{CD}$ in family, presence of AIDs in family, smoking and alcohol use, educational status, marital status, Body Mass Index (BMI)) of the patients included in the study were documented. Height (meter) and weight $(\mathrm{kg})$ measurements were made to calculate the BMI of the patients. The BMI was calculated with the following formula: Weight/Height $x$ Height. The waist circumference, hip circumference, waist/hip rates were also documented. In men, 0.9 waist/hip rate was considered to be risk for abdominal obesity and chronic diseases in men, and 0.85 in women. ${ }^{11}$ The symptoms of the participants at admission were documented. All participants were asked to fill in a questionnaire to evaluate the symptoms that were related with the gastrointestinal system. The presence of diarrhea, constipation, reflux, dyspepsia, abdominal pain, nausea, and vomiting were analyzed in patients in the questionnaire. ${ }^{12}$ In addition to the GIS symptoms, the presence of anemia, thyroid diseases, weight loss, growth retardation, infertility, skin lesions, bleeding complaints, bone disorders, and extrahepatic cholestasis were also documented. The antibody levels were evaluated again at the last visit. The patients who had negative antibody levels were accepted to be in immunological remission.

Dietary Compliance: In patients who had CD, gluten-free diet compliance was questioned retrospectively in the patient records in patient visits by the responsible physician and dietician. In addition, compliance with gluten-free nutrition was evaluated by analyzing the questionnaire that was given to the patients. The diet was classified as two categories; 1 ) Strict diet (complete dietary compliance), 2) Normal gluten-containing diet and partial dietary compliance.

Autoimmune Diseases: The presence of AIDs in celiac patients was determined with a structured questionnaire form and patient records (AIDs were diagnosed with anamnesis, physical examination findings, laboratory and imaging methods). The questionnaire was filled by the doctor during the visit of the patient. The presence of AIDs was questioned in all subjects with a predetermined list. IDDM, autoimmune thyroid diseases, autoimmune hepatitis, primary biliary cirrhosis, dermatitis herpetiormis, collagen tissue diseases, 
immune thrombocytopenia, asthma, autoimmune hypoparathyrodism, autoimmune hypogonadism, and skin diseases were assessed separately. (Dated November 11, 2014) was obtained from the Ethics Committee of our hospital. All the applied procedures were complied with the ethical standards of human testing committee of our institution and the Helsinki Declaration.

Data Analysis: The results of our study were analyzed with "the Statistical Package for Social Sciences 19.0 (SPSS Armonk, NY: IBM Corp.)" program. The data that had continuous values were given as (mean \pm standard deviation), and the categorical data were given as frequency and percentage $(n, \%)$. The data were tested for compliance to normal distribution with the Kolmogorov-Simirnov Test, Histogram and \pm SD. The nonparametric data of the groups were compared by using the Mann Whitney U-test; and the parametric data were compared with the Parametric T-test. The Chi-square Test was employed to test the categorical data. In multivariate analysis, the independent predictors were evaluated by using the Logistic Regression Analysis to predict the AID development related to the $C D$ by using possible factors that were determined in previous analyzes. A P-value $<0.05$ was considered to be statistically significant.

\section{RESULTS}

A total of 230 patients who were followed-up with CD were included in the study. A total of $172(74.8 \%)$ of the patients were female, and the mean age was $35.6 \pm 10.6$ years (range between 1872 years). The mean age of onset of disease was $29.1 \pm 6.5$ years (range between $4-66$ years). The disease duration was between 1-29 years, and the mean duration was $6.55 \pm 4.6$ years. The mean BMI level of the patients was 22.1 \pm 4.1 , waist-hip ratio of the patients ranged between 0.63 and 0.98 with a mean of $0.81 \pm 0.07$. The waist-hip rate was $0.87 \pm 0.06$ in males, and $0.8 \pm 0.06$ in females. In $46(20 \%)$ of the patients, it was determined that the waist-hip rate was above normal. According to the March classification, when stage 1, 2 and 3a were evaluated as mild and stage $3 b, 3 c$ and stage 4 were evaluated as advanced disease, it was determined that $101(43.9 \%)$ patients were mild, and $129(56.1 \%)$ patients were in severe stage.

Evaluation for AIDs revealed that; 72(31.3\%) of the 230 patients had AIDs. A total of $45(19.6 \%)$ of these patients had only one AIDs, 18(7.8\%) had two AIDs, and 9(3.9\%) had three and more AIDs. Hashimoto thyroiditis was determined in $39(17 \%)$ patients, asthma in $16(7 \%)$ patients, IDDM in $10(4.3 \%)$ patients, dermatitis herpetiformis and autoimmune hepatitis in $8(3.5 \%)$ patients each, Sjogren in $6(2.6 \%)$ patients, autoimmune hypoparathyrodism in $4(1.7 \%)$ patients, immune thrombocytopenia in 3(1.3\%) patients, Grave's disease, primary biliary cirrhosis, psoriasis, Behcet's disease, scleroderma, dermatomyositis, familial mediterranean fever, and rheumatoid arthritis in $2(0.9 \%)$ patients each, systemic lupus erythematosus, gout disease, autoimmune hypogonadism and ankylosing spondylitis in 1 patient $(0.45 \%)$ each (Table-I).

The factors that might affect the development of AIDs in celiac patients were evaluated. There was a statistically significant relation between AIDs development and female gender, age of diagnosis under 40 years, long duration of illness, presence of non-GIS symptoms at the time of first admission and working status. Although the AIDs development was less in patients who had strict diet; however, this was not statistically significant. No significant relations were detected between AID development and other risk factors. While the rate of being young (below 40 years) was $87.5 \%$ in individuals with autoimmune disease accompanying $\mathrm{CD}$, this

Table-I: Accompanying autoimmune diseases in celiac patients.

\begin{tabular}{llll}
\hline & $N-(\%)$ & & $N-(\%)$ \\
\hline Dermatitis Herpetiformis & $8(3.5 \%)$ & Rheumatoid arthritis & $2(0.9 \%)$ \\
Type 1 Diabetes Mellitus & $10(4.3 \%)$ & Asthma & $16(7 \%)$ \\
Hashimoto Thyroiditis & $39(17 \%)$ & Immune Thrombocytopenia & $3(1.3 \%)$ \\
Graves' Disease & $2(0.9 \%)$ & Hypogonadism & $1(0.45 \%)$ \\
Primer Biliary Cirrhosis & $2(0.9 \%)$ & Sjogren & $6(2.6 \%)$ \\
Autoimmune Hepatitis & $8(3.5 \%)$ & Psoriasis & $2(0.9 \%)$ \\
Systemic Lupus Erythematosus & $1(0.45 \%)$ & Autoimmune Hypoparathyroidism & $4(1.7 \%)$ \\
Scleroderma & $2(0.9 \%)$ & Dermatomyositis & $2(0.9 \%)$ \\
Familial Mediterranean Fever & $2(0.9 \%)$ & Gout Disease & $1(0.45 \%)$ \\
Behcet's Disease & $2(0.9 \%)$ & Ankylosing Spondylitis & $1(0.45 \%)$ \\
\hline
\end{tabular}

N: Number of patients. 
rate was $75.3 \%$ in individuals without autoimmune disease accompanying $C D(p=0.035)$. The duration of $\mathrm{CD}$ in individuals who had AID accompanying $C D$ was $7.92 \pm 5$, and the duration of disease in individuals who did not have AID was 5.92 \pm 4.3 $(p=0.002)$. Only $70.9 \%$ of individuals without CD associated AID were females, however $83.3 \%$ of individuals with $\mathrm{CD}$ associated AID were females $(p=0,044)$. Only $74.1 \%$ of of individuals without
CD associated AID were not working, however $87.5 \%$ of individuals with CD associated AID were not working $(p=0,022)$. While $20.9 \%$ of individuals without $C D$ associated AID had non-GIS symptoms, $34.7 \%$ of individuals with CD associated AID had non-GIS symptoms $(\mathrm{p}=0.025)$ (Table-II).

The logistic regression model that included factors like having GIS symptoms at admission, the age of onset of $\mathrm{CD}$ being $<40$ years, who had

Table-II: Risk factors associated with the presence of autoimmune disease.

\begin{tabular}{|c|c|c|c|c|}
\hline Variables & $\begin{array}{l}\text { Celiac Disease without } \\
\text { Autoimmune Disease }\end{array}$ & $\begin{array}{l}\text { Celiac Disease with } \\
\text { Autoimmune Disease }\end{array}$ & Total & P value \\
\hline Age (years \pm SS) & $35.9 \pm 12.07$ & $34.9 \pm 10.7$ & $35.6 \pm 10.6$ & 0.57 \\
\hline Onset age of the disease (years) & $30 \pm 11.7$ & $27 \pm 10.4$ & $29.1 \pm 6.5$ & 0.09 \\
\hline \multicolumn{5}{|l|}{ Onset age of the disease (years) } \\
\hline$<40$ years of age & $119(75.3 \%)$ & $63(87.5 \%)$ & $182(79.2 \%)$ & $0.035^{*}$ \\
\hline$\geq 40$ years of age & $39(24.7 \%)$ & $9(12.5 \%)$ & $48(20.8 \%)$ & \\
\hline Disease duration (years) & $5.92 \pm 4.3$ & $7.92 \pm 5$ & $6.55 \pm 4.6$ & $0.002^{*}$ \\
\hline BMI $\left(\mathrm{kg} / \mathrm{m}^{2}\right)$ & $22 \pm 4$ & $22.4 \pm 3.9$ & $22.1 \pm 4.1$ & 0.49 \\
\hline \multicolumn{5}{|l|}{ Waist/hip rate } \\
\hline Below the limit & $127(80.4 \%)$ & $57(79.2 \%)$ & $184(80 \%)$ & 0.83 \\
\hline Above the limit & $31(19.6 \%)$ & $15(20.8 \%)$ & $46(20 \%)$ & \\
\hline \multicolumn{5}{|l|}{ Disease stage } \\
\hline Mild (Stage 2-3a) & $74(46.8 \%)$ & $27(37.5 \%)$ & $101(43.9 \%)$ & 0.18 \\
\hline Severe (Stage 3b,3c,4) & $84(53.2 \%)$ & $45(62.5 \%)$ & $129(56.1 \%)$ & \\
\hline \multicolumn{5}{|l|}{ Dietary Compliance } \\
\hline Strict & $90(57 \%)$ & $32(44.4 \%)$ & $122(53.1 \%)$ & 0.078 \\
\hline None & $68(43 \%)$ & $40(55.6 \%)$ & $108(46.9 \%)$ & \\
\hline \multicolumn{5}{|l|}{ Gender } \\
\hline Female & $112(70.9 \%)$ & $60(83.3 \%)$ & $172(74.7 \%)$ & $0.044^{*}$ \\
\hline Male & $46(29.1 \%)$ & $12(16.7 \%)$ & $58(25.3 \%)$ & \\
\hline \multicolumn{5}{|l|}{ Immunologic Remission } \\
\hline Yes & $70(44.3 \%)$ & $38(52.8 \%)$ & $108(46.9 \%)$ & 0.23 \\
\hline No & $88(55.7 \%)$ & $34(47.2 \%)$ & $122(53.1 \%)$ & \\
\hline \multicolumn{5}{|l|}{ Family History } \\
\hline Yes & $39(24.7 \%)$ & $11(15.3 \%)$ & $50(21.7 \%)$ & 0.10 \\
\hline No & $119(75.3 \%)$ & $61(84.7 \%)$ & $180(78.3 \%)$ & \\
\hline \multicolumn{5}{|l|}{ Educational Status } \\
\hline Illiterate & $30(19 \%)$ & $16(22.2 \%)$ & $46(20 \%)$ & 0.38 \\
\hline Primary Education & $87(55.1 \%)$ & $37(51.4 \%)$ & $124(53.9 \%)$ & \\
\hline High School and over & $41(25.9 \%)$ & $19(26.4 \%)$ & $60(26.1 \%)$ & \\
\hline \multicolumn{5}{|l|}{ Marital Status } \\
\hline Married & $114(72.2 \%)$ & $50(69.4 \%)$ & $164(71.3 \%)$ & 0.67 \\
\hline Single & $44(27.8 \%)$ & $22(30.6 \%)$ & $66(28.7 \%)$ & \\
\hline \multicolumn{5}{|l|}{ Working Status } \\
\hline Working & $41(25.9 \%)$ & $9(12.5 \%)$ & $50(21.7 \%)$ & $0.022^{*}$ \\
\hline Unemployed & $117(74.1 \%)$ & $63(87.5 \%)$ & $180(78.3 \%)$ & \\
\hline \multicolumn{5}{|l|}{ Smoking Status } \\
\hline Smoking & $26(16.5 \%)$ & $18(25 \%)$ & $44(19.1 \%)$ & 0.20 \\
\hline Non-smoking & $116(73.4 \%)$ & $50(69.4 \%)$ & $166(72.2 \%)$ & \\
\hline Quit & $16(10.1 \%)$ & $4(5.6 \%)$ & $20(8.7 \%)$ & \\
\hline \multicolumn{5}{|l|}{ Complaints at First Admission } \\
\hline GIS symptoms & $125(79.1 \%)$ & $47(65.3 \%)$ & $172(74.8 \%)$ & $0.025^{*}$ \\
\hline Non-GIS symptoms & $33(20.9 \%)$ & $25(34.7 \%)$ & $58(25.2 \%)$ & \\
\hline
\end{tabular}

BMI: Body Mass Index, GIS: Gastrointestinal System * : Statistically significant $(\mathrm{p}<0.05)$. 
a history of $\mathrm{CD}$ in the family, having immune remission, gender, working status, having non-GIS symptoms at first admission were used to examine the independent risk factors that determined presence of AIDs accompanied CD. As a result of the analysis, it was determined that the following were independent risk factors in AID development; having non-GIS symptoms at first admission (OR:2.29, 95\% CI:1.195-4.388, $\mathrm{p}=0.013$ ); the age of onset of the disease being <40 years (OR: 2.299, 95\% CI:1.030-5.131, $\mathrm{p}=0.042)$; and unemployment of the patient (OR: 2.928, 95\% CI:1.195-4.388, $\mathrm{p}=0.013$ ).

\section{DISCUSSION}

In previous studies, it was shown that; there is a significant increase in the prevalence of AIDs in celiac patients and their first-degree relatives when compared to the normal population. ${ }^{3}$ Especially, since type 1 diabetes mellitus and hashimoto's thyroiditis often accompany $\mathrm{CD}$. For that, routine screening of these AIDs is recommended in all patients with CD. ${ }^{13,14}$ Ventura and Viljama conducted a study and showed that the AIDs prevalence was $34 \%$ and $31 \%$ in celiac patient. They also showed that thyroid diseases, IDDM, asthma and skin diseases accompanied CD frequently., ${ }^{5,6}$ Bibbo et al. conducted a study and determined an AID in the control group at a rate of $15.2 \%$. They also showed that this rate was $35.3 \%$ in CD patients. ${ }^{15}$ Demirezer Bolat et al. conducted a study with 145 patients who had $C D$, and reported that $33.1 \%$ of the patients had accompanying AIDs to CD; and that the following were the most frequent accompanying diseases: hashimoto thyroiditis $(24.1 \%)$, type 1 diabetes mellitus (5.5\%) and psoriasis $(2.8 \%) .{ }^{16}$ These data show similarities with the findings of our study.

In our study in which 230 celiac patients were included, it was determined that 72 (31.3\%) patients had AIDs, and that $39(17 \%)$ patients had hashimoto thyroiditis, $16(7 \%)$ patients had asthma, and $10(4.3 \%)$ patients had type 1 diabetes mellitus as the most common AIDs. It was reported that the pathophysiological basis of the relation between CD and AIDs was sharing of HLA-DR3, HLA-DQ2 and other genetic loci that played roles in AID development. ${ }^{17,18}$ In addition, exposure of an immature immune system to gliadin in sensitive individuals causes an immune response modification at an early time, and as a result, an AID together with CD. For these reasons, it is considered that the AID risk is high in celiac patients. ${ }^{19}$

Many environmental factors play roles in the basis of $C D$ and other AID in addition to genetic characteristics. Viljama et al. conducted a study and showed that there was a significant relation between early diagnosis of $\mathrm{CD}$, disease duration, female gender, long duration of gluten exposure, and coexistence of AID. ${ }^{6}$ Similarly, Cosnes et al. conducted another study and found a significant relation between presence of AIDs in family, presence of non-GIS symptoms in patients at admission, and development of AID in patients with diagnosis of $\mathrm{CD}$ at age $<36$ years. ${ }^{7}$ Bibbo et al. also showed that long disease duration is a predictive factor in AID development. ${ }^{15}$ In the present study of ours, it was found that the age of disease diagnosis being $<40$ years of age, non-GIS symptoms at first admission, and unemployment of the patient were independent risk factors for AID development.

However, it was observed that the reported data are contradictory in studies examining the relation between gluten-free diet adaptation and AID development. Some studies showed that organ-specific antibodies were more prevalent in celiac patients who were not treated, and gluten exposure time was a predisposing factor in AID development. ${ }^{5,20}$ Ventura et al. ${ }^{5}$ showed that organspecific antibodies were reduced at a significant level in patients who meet gluten-free dietary therapy and that gluten-free diet prevents the development of AID. However, in some previous studies, it was argued that applying gluten-free diet therapy does not prevent AID development; however, in some other studies, it was reported gluten-free diet therapy decreases AID development, yet, it did not provide total protection. ${ }^{6,7,10}$ Also, Ouaka-Kchaou et al. showed that duration of the disease was longer at a significant level in $\mathrm{CD}$ patient accompanied by AID. They also determined that strict dietary compliance was $46 \%$ in the AID-accompanied group, and $67 \%$ in the group without AID. ${ }^{21}$

In the present study of ours, although it was determined that there were no significant relation between strict dietary compliance and AID development, it was found that diet adaptation was less in the group in which AIDs accompanied CD. In addition, we believe that; the significant relation between prolonged disease duration and AID development suggest that longer exposure to gluten of celiac patients increases the risk of AID development (due to non-compliance to diet).

In Turkey, there are no comprehensive studies that examine the risk factors related to AID and development of the accompanying diseases in a large population. Since the patients who were followed in polyclinics of important healthcare 
center receiving patients from all surrounding cities were included in our study, a large geographic population was analyzed in the present study. However, there are some limitations of our study. Firstly, although all patients were interviewed face-to-face, and all the data were checked for accuracy, the data were collected retrospectively. Secondly, the patients who did not have clinically obvious complaints; however, who could have subclinical AIDs were not included in the present study. Another limitation of the present study of ours is that; it was not possible to predict the amount of gluten patients took in the long term in an accurate manner. The patients' remission status was evaluated as clinical laboratory and immunological. However, due to the lack of an objective measurement method for the amount of gluten taken, dietary compliance was determined by using a questionnaire filled with dietician and using the information obtained from the registration data.

\section{CONCLUSION}

In conclusion, our study has shown that; it is important to screen other AIDs in celiac patients, especially in those who have risk factors. It must be kept in mind that patients who present with GIS symptoms must be screened in terms of $C D$, and also, patients who present with nonGIS complaints may have CD, and that AIDs accompanies $\mathrm{CD}$ more in patients presenting with these complaints. Because of the presence of many AIDs and extra-intestinal involvements in celiac patients, these patients must be treated in an integrity with a multidisciplinary approach.

\section{Conflicting Interests: None.}

Financial Disclosures: None.

\section{REFERENCES}

1. Rubio-Tapia A, Hill ID, Kelly CP, Calderwood AH, Murray JA. ACG clinical guidelines: diagnosis and management of celiac disease. Am J Gastroenterol. 2013;108(5):656. doi: 10.1038/ajg.2013.79

2. Di Sabatino A, Corazza GR. Coeliac disease. Lancet. 2009;373(9673):1480-1493. doi: 10.1016/S01406736(09)60254-3

3. Zanoni G, Navone R, Lunardi C, Tridente G, Bason C, Sivori S, et al. In celiac disease, a subset of autoantibodies against transglutaminase binds toll-like receptor 4 and induces activation of monocytes. PLoS Med. 2006;3(9):358. doi:10.1371/journal.pmed.0030358

4. Alaedini A, Green HR, Narrative Review: Celiac Disease: Understanding a Complex Autoimmune Disorder. Ann Intern Med. 2005;142:289-298. doi:10.7326/0003-4819-142-4200502150-00011
5. Ventura A, Magazzù G, Greco L. Duration of exposure to gluten and risk for autoimmune disorders in patients with celiac disease. Gastroenterology. 1999;117(2):297-303. doi:10.1053/gast.1999.0029900297

6. Viljamaa M, Kaukinen $\mathrm{K}$, Huhtala $\mathrm{H}$, Kyrönpalo $\mathrm{S}$, Rasmussen M, Collin P. Coeliac disease, autoimmune diseases and gluten exposure. Scand J Gastroenterol. 2005;40(4):437-443. doi:10.1080/00365520510012181

7. Cosnes J, Cellier C, Viola S, Colombel JF, Michaud L, Sarles $\mathrm{J}$, et al. Incidence of autoimmune diseases in celiac disease: protective effect of the gluten-free diet. Clin Gastroenterol Hepatol. 2008;6(7):753-758. doi: 10.1016/j.cgh.2007.12.022

8. Sollid LM, Jabri B. Is celiac disease an autoimmune disorder? Curr Opin Immunol. 2005;17(6):595-600. doi:10.1016/j. coi.2005.09.015

9. Ventura A, Neri E, Ughi C, Leopaldi A, Citta A, Not T. Gluten-dependent diabetes-related and thyroid-related autoantibodies in patients with celiac disease. J Pediatr. 2000;137:263-265. doi:10.1067/mpd.2000.107160

10. Sategna Guidetti C, Solerio E, Scaglione N, Aimo G, Mengozzi G. Duration of gluten exposure in adult coeliac disease does not correlate with the risk of autoimmune disorders. Gut. 2001;49:502-505. doi:10.1136/gut.49.4.502

11. American Diabetes Association. Standards of Medical Care in Diabetes. Diabetes Care. 2015;38:8-67. doi: 10.2337/dc15-S003

12. Revicki DA, Wood M, Wiklund I, Crawley J. Reliability and validity of the Gastrointestinal Symptom Rating Scale in patients with gastroesophageal reflux disease. Qual Life Res. 1998;7(1):75-83. doi: 10.1023/ A:1008841022998

13. Ludvigsson JF, Bai JC, Biagi F, Card TR, Ciacci C, Ciclitira PJ, et al. Diagnosis and management of adult coeliac disease: guidelines from the British Society of Gastroenterology. Gut. 2014;63:1210-1228. doi: 10.1136/gutjnl-2013-306578

14. Rubio-Tapia A, Hill ID, Kelly CP, Calderwood AH, Murray JA. American College of G. ACG clinical guidelines: diagnosis and management of celiac disease. Am J Gastroenterol. 2013;108:656-676. doi: 10.1038/ajg.2013.79

15. Bibbò S, Pes GM, Usai-Satta P, Salis R, Soro S, Quarta Colosso $\mathrm{BM}$, et al. Chronic autoimmune disorders are increased in coeliac disease: A case-control study. Medicine (Baltimore). 2017;96(47):8562. doi: 10.1097/MD.0000000000008562

16. Demirezer Bolat A, Akin FE, Tahtaci M, Tayfur Yürekli Ö, Köseoğlu H, Erten Ş, et al. Risk factors for polyautoimmunity among patients with celiac disease: a cross-sectional survey. Digestion. 2015;92:185-191. doi: 10.1159/000439586

17. Smyth DJ, Plagnol V, Walker NM, Cooper JD, Downes K, Yang JH, et al. Shared and distinct genetic variants in type 1 diabetes and celiac disease. N Engl J Med. 2008;359:27672677. doi: 10.1056/NEJMoa0807917

18. Fallahi P, Ferrari SM, Ruffilli I, Elia G, Biricotti M, Vita R, et al. The association of otherautoimmune diseases in patients with autoimmune thyroidiitis: review ofthe literature and report of a large series of patients. Autoimmun Rev. 2016;15:1125-1128. doi: 10.1016/j.autrev.2016.09.009

19. Ventura A, Magazù G, Gerarduzzi T, Greco L. Coeliac disease and the risk of autoimmune disorders. Gut. 2002;51:897-898. doi:10.1136/gut.51.6.897

20. Toscano V, Conti F.G, Anastasi E, Mariani P, Tiberti C, Poggi $\mathrm{M}$, et al. Importance of gluten in the induction of endocrine autoantibodies and organ dysfunction in adolescent celiac patients, Am J Gastroenterol. 2000;95:1742-1748. do1:10.1111/j.1572-0241.2000.02187.x

21. Ouaka-Kchaou A, Ennaifer R, Elloumi H, Gargouri D, Hefaiedh R, Kochlef A et al. Autoimmune diseases in coeliac disease: effect of gluten exposure. Therap Adv Gastroenterol. 2008;1(3):169-172. doi: 10.1177/1756283X08096612 